\title{
ON A NON-CONVEX HYPERBOLIC DIFFERENTIAL INCLUSION
}

\author{
by VASILE STAICU
}

(Received 16th April 1990)

\begin{abstract}
We prove the existence of a solution $u(., . ; \alpha, \beta)$ of the Darboux problem $u_{x y} \in F(x, y, u), u(x, 0)=\alpha(x)$, $u(0, y)=\beta(y)$, which is continuous with respect to $(\alpha, \beta)$. We assume that $F$ is Lipschitzean with respect to $u$ but not necessarily convex valued.
\end{abstract}

1980 Mathematics subject classification (1985 Revision): 34A60, 35B30.

\section{Introduction and main result}

Let $I=[0,1], Q=I \times I$ and denote by $\mathscr{L}$ the $\sigma$-algebra of the Lebesgue measurable subsets of $Q$. Denote by $2^{R^{n}}$ the family of all closed nonempty subsets of $R^{n}$ and by $\mathscr{B}\left(R^{n}\right)$ the family of all Borel subsets of $R^{n}$. For $x \in R^{n}$ and $A, B \in 2^{R^{n}}$ we denote by $d(x, A)$ the usual point-to-set distance from $x$ to $A$ and by $h(A, B)$ the Hausdorff pseudodistance from $A$ to $B$.

By $C\left(Q, R^{n}\right)$ (resp. $L^{1}\left(Q, R^{n}\right)$ ) we denote the Banach space of all continuous (resp. Bochner integrable) functions $u: Q \rightarrow R^{n}$ with the norm $\|u\|_{\infty}=\sup \{\|u(x, y)\|:(x, y) \in Q\}$ (resp. $\left.\|u\|_{1}=\int_{0}^{1} \int_{0}^{1}\|u(x, y)\| d x d y\right)$, where $\|$.$\| is the norm in R^{n}$.

Recall that a subset $K$ of $L^{1}\left(Q, R^{n}\right)$ is said to be decomposable ([9]) if for every $u$, $v \in K$ and $A \in \mathscr{L}$ we have $u \chi_{A}+v \chi_{Q \backslash A} \in K$, where $\chi_{A}$ stands for the characteristic function of $A$. We denote by $\mathscr{D}$ the family of all decomposable closed nonempty subsets of $L^{1}\left(Q, R^{n}\right)$.

Let $F: Q \times R^{n} \rightarrow 2^{R^{n}}$ be a multivalued map. Recall that $F$ is called $\mathscr{L} \otimes$ $\mathscr{B}\left(R^{n}\right)$-measurable if for any closed subset $C$ of $R^{n}$ we have that $\{(x, y, z) \in Q \times$ $\left.R^{n}: F(x, y, z) \cap C \neq \varnothing\right\} \in \mathscr{L} \otimes \mathscr{B}\left(R^{n}\right)$.

We associate to $F: Q \times R^{n} \rightarrow 2^{R^{n}}$ the Darboux problem

$$
u_{x y} \in F(x, y, u), \quad u(x, 0)=\alpha(x), \quad u(0, y)=\beta(y),
$$

where $\alpha, \beta$ are two continuous functions from $I$ into $R^{n}$ with $\alpha(0)=\beta(0)$.

Definition. $u(., . ; \alpha, \beta) \in C\left(Q, R^{n}\right)$ is said to be a solution of the Darboux problem $\left(D_{\alpha \beta}\right)$ if there exists $v(., . ; \alpha, \beta) \in L^{1}\left(Q, R^{n}\right)$ such that 
(i) $v(x, y ; \alpha, \beta) \in F(x, y, u(x, y ; \alpha, \beta))$ a.e. in $Q$,

(ii) $u(x, y ; \alpha, \beta)=\alpha(x)+\beta(y)-\alpha(0)+\int_{0}^{x} \int_{0}^{y} v(\xi, \eta ; \alpha, \beta) d \xi d \eta$, for every $(x, y) \in Q$.

Note that the function $v(., . ; \alpha, \beta)$ which corresponds to $u(., . ; \alpha, \beta)$ in the above definition is unique (a.e.). Consider the Banach space

$$
\mathbf{S}=\left\{(\alpha, \beta) \in C\left(I, R^{n}\right) \times C\left(I, R^{n}\right): \alpha(0)=\beta(0)\right\}
$$

endowed with the norm

$$
\|(\alpha, \beta)\|=\|\alpha\|_{\infty}+\|\beta\|_{\infty}
$$

and, for $(\alpha, \beta)$ in $\mathbf{S}$, we denote by $\mathbf{T}(\alpha, \beta)$ the set of all solutions of the problem $\left(D_{\alpha \beta}\right)$. The aim of this note is to prove the following:

Theorem. Let $F: Q \times R^{n} \rightarrow 2^{R^{n}}$ satisfy the following assumptions:

$\left(H_{1}\right) \quad F$ is $\mathscr{L} \otimes \mathscr{B}\left(R^{n}\right)$-measurable,

$\left(H_{2}\right)$ there exists $L>0$ such that $h(F(x, y, u), F(x, y, v)) \leqq L\|u-v\|$ for all $u, v \in R^{n}$, a.e. in $Q$,

$\left(H_{3}\right)$ there exists a function $\delta \in L^{1}(Q, R)$ such that $d(0, F(x, y, 0)) \leqq \delta(x, y)$ a.e. in $Q$.

Then there exists $u: Q \times \mathbf{S} \rightarrow R^{n}$ such that

(i) $u(., . ; \alpha, \beta) \in \mathbf{T}(\alpha, \beta)$ for every $(\alpha, \beta) \in \mathbf{S}$

(ii) $(\alpha, \beta) \rightarrow u(., . ; \alpha, \beta)$ is continuous from $\mathbf{S}$ to $C\left(Q, R^{n}\right)$.

In other words we prove the existence of a global solution $u(., . ; \alpha, \beta)$ of the problem $\left(D_{\alpha \beta}\right)$ depending continuously on $(\alpha, \beta)$ in the space $\mathbf{S}$.

This result is a natural extension of the well posedness property (i.e., existence of a unique solution depending continuously on the initial data) of the Darboux problems defined by Lipschitzean single-valued maps (see [3]). We obtain the solution by a completeness argument without assumptions on the convexity or boundedness of the values of $F$.

Filippov has obtained in [7] the existence of solutions to an ordinary differential inclusion $x^{\prime} \in F(t, x)$ defined by a multifunction $F$ Lipschitzian with respect to $x$, without assumptions on the convexity or boundedness of the values $F(t, x)$ by using a successive approximation process.

Following an idea in [4] we extend this process to Darboux problems and we do it continuously with respect to $(\alpha, \beta)$ in the space $\mathbf{S}$ by using a result on the existence of a continuous selection from multifunctions with decomposable values, proved in [8] and extended in [2].

The construction in the proof of our theorem works for the case when $F$ is Lipschitzian in $u$, but the assumption $\left(\mathrm{H}_{2}\right)$ is not only a technical one. We shall give an example showing that if $\left(\mathrm{H}_{2}\right)$ is relaxed, allowing $F$ to be merely continuous then the conclusion of the theorem is in general no longer true. 
However the Lipschitz property of $F$ is not necessary for the existence of solutions. If $F$ is upper semicontinuous with compact convex values then the existence of local and global solutions has been obtained in [11] and [12], by using the Kakutani-Ky Fan fixed point theorem. The convexity assumption is essential in this case. To avoid the convexity assumption we have to increase the regularity of $F$. If $F$ is a Caratheodory function which is compact not necessarily convex valued then there exists a solution of the Darboux problem and this fact has been proved in [13] by using a continuous selection argument and the Schauder fixed point theorem. Qualitative properties and the structure of the set of solutions of Darboux problems has been studied in [5] and [6].

Remark finally that another extension of the well posedness property of a Darboux problem defined by a multifunction Lipschitzian in $u$, lower semicontinuous with respect to a parameter, expressed in terms of lower semicontinuous dependence of the set of all solutions of the problem on the initial data and parameter is given in [10].

\section{Proof of the main result}

In the following two lemmas $S$ is a separable metric space. Let $X$ be a Banach space and $G: S \rightarrow 2^{X}$ be a multifunction. Recall that $G$ is said to be lower semicontinuous (l.s.c.) if for every closed subset $C$ of $X$ the set $\{s \in S: G(s) \subset C\}$ is closed in $S$.

Lemma 1 ([4, Proposition 2.1]). Assume $F_{*}: Q \times S \rightarrow 2^{R^{n}}$ to be $\mathscr{L} \otimes \mathscr{B}(S)$-measurable, l.s.c. with respect to $s \in S$. Then the map $s \rightarrow G_{*}(s)$ given by

$$
G_{*}(s)=\left\{v \in L^{1}\left(Q, R^{n}\right): v(x, y) \in F_{*}(x, y, s) \text { a.e. in } Q\right\}, \quad s \in S,
$$

is l.s.c. with decomposable closed nonempty values if and only if there exists a continuous function $\sigma: S \rightarrow L^{1}(Q, R)$ such that $d\left(0, F_{*}(x, y, s)\right) \leqq \sigma(s)(x, y)$ a.e. in $Q$.

Lemma 2 ([4, Proposition 2.2]). Let $G: S \rightarrow \mathscr{D}$ be a l.s.c. multifunction and let $\phi: S \rightarrow L^{1}\left(Q, R^{n}\right)$ and $\psi: S \rightarrow L^{1}(Q, R)$ be continuous maps. If for every $s \in S$ the set

$$
H(\xi)=c l\{v \in G(s):\|v(x, y)-\phi(s)(x, y)\|<\psi(s)(x, y) \text { a.e. in } Q\}
$$

is nonempty then the map $H: S \rightarrow \mathscr{D}$ defined by (2.1) admits a continuous selection.

We note that the second lemma is a direct consequence of Proposition 4 and Theorem 3 in [2] (see also [8]).

Proof of the theorem. Fix $\varepsilon>0$ and set $\varepsilon_{n}=\varepsilon / 2^{n+1}, n \in N$. For $(\alpha, \beta) \in \mathbf{S}$ define $u_{0}(., ; \alpha, \beta): Q \rightarrow R^{n}$ by $u_{0}(x, y ; \alpha, \beta)=\alpha(x)+\beta(y)-\alpha(0)$ and observe that for all $(x, y) \in Q$ we have

$$
\left\|u_{0}\left(x, y ; \alpha_{1}, \beta_{2}\right)-u_{0}\left(x, y ; \alpha_{2}, \beta_{2}\right)\right\| \leqq\left\|\alpha_{1}(x)-\alpha_{2}(x)\right\|+\left\|\beta_{1}(y)-\beta_{2}(y)\right\|+\left\|\alpha_{1}(0)-\alpha_{2}(0)\right\|
$$




$$
\leqq 2\left\|\left(\alpha_{1}, \beta_{1}\right)-\left(\alpha_{1}, \beta_{2}\right)\right\| \text {. }
$$

This implies that $(\alpha, \beta) \rightarrow u_{0}(., . ; \alpha, \beta)$ is continuous from $\mathbf{S}$ to $C\left(Q, R^{n}\right)$. Setting $\sigma(\alpha, \beta)(x, y)=\delta(x, y)+L\left\|u_{0}(x, y ; \alpha, \beta)\right\|$ we obtain that $\sigma$ is a continuous map from $\mathbf{S}$ to $L^{1}(Q, R)$ and

$$
d\left(0, F\left(x, y, u_{0}(x, y ; \alpha, \beta)\right)\right) \leqq \sigma(\alpha, \beta)(x, y) \text { a.e. in } Q
$$

For $(\alpha, \beta) \in \mathbf{S}$, define

$$
G_{0}(\alpha, \beta)=\left\{v \in L^{1}(Q, X): v(x, y) \in F\left(x, y, u_{0}(x, y ; \alpha, \beta)\right) \text { a.e. in } Q\right\},
$$

and

$$
H_{0}(\alpha, \beta)=c l\left\{v \in G_{0}(\alpha, \beta):\|v(x, y)\|<\sigma(\alpha, \beta)(x, y)+\varepsilon_{0} \text { a.e. in } Q\right\} .
$$

Then, by (2.2) and Lemma 1, it follows that $G_{0}$ is 1.s.c. from $\mathbf{S}$ into $\mathscr{D}$ and, by (2.2), $H_{0}(\alpha, \beta) \neq \varnothing$ for each $(\alpha, \beta) \in \mathbf{S}$. Therefore by Lemma 2 , there exists $h_{0}: \mathbf{S} \rightarrow L^{1}\left(Q, R^{n}\right)$, which is a continuous selection of $H_{0}$. Set $v_{0}(x, y ; \alpha, \beta)=h_{0}(\alpha, \beta)(x, y)$ and observe that $v_{0}(x, y ; \alpha, \beta) \in F\left(x, y, u_{0}(x, y ; \alpha, \beta)\right)$ and $\left\|v_{0}(x, y)\right\| \leqq \sigma(\alpha, \beta)(x, y)+\varepsilon_{0}$, for a.e. $(x, y) \in Q$. Define

$$
u_{1}(x, y ; \alpha, \beta)=u_{0}(x, y ; \alpha, \beta)+\int_{0}^{x} \int_{0}^{y} v_{0}(\xi, \eta ; \alpha, \beta) d \xi d \eta
$$

and, for $n \geqq 1$, set

$$
\sigma_{n}(\alpha, \beta)(x, y)=L^{n-1}\left[\int_{0}^{x} \int_{0}^{y} \frac{(x-\xi)^{n-1}}{(n-1) !} \frac{(y-\eta)^{n-1}}{(n-1) !} \sigma(\alpha, \beta)(\xi, \eta) d \xi d \eta+\left(\sum_{i=0}^{n} \varepsilon_{i}\right) \frac{(x+y)^{n}}{n !}\right]
$$

Then, for every $(x, y) \in Q \backslash\{0,0)\}$, we have

$$
\begin{aligned}
\left\|u_{1}(x, y ; \alpha, \beta)-u_{0}(x, y ; \alpha, \beta)\right\| & \leqq \int_{0}^{x} \int_{0}^{y}\left\|v_{0}(\xi, \eta ; \alpha, \beta)\right\| d \xi d \eta \leqq \int_{0}^{x} \int_{0}^{y} \sigma(\alpha, \beta)(\xi, \eta) d \xi d \eta+\varepsilon_{0}(x+y) \\
& <\sigma_{1}(\alpha, \beta)(x, y),
\end{aligned}
$$

and so

$$
d\left(v_{0}(x, y ; \alpha, \beta), F\left(x, y, u_{1}(x, y ; \alpha, \beta)\right) \leqq L\left\|u_{1}(x, y ; \alpha, \beta)-u_{0}(x, y ; \alpha, \beta)\right\|<L \sigma_{1}(\alpha, \beta)(x, y) .\right.
$$

We claim that there exist two sequences $\left\{v_{n}(x, y ; \alpha, \beta)\right\}_{n \in N}$ and $\left\{u_{n}(x, y ; \alpha, \beta)\right\}_{n \in N}$ such that for each $n \geqq 1$ we have:

(a) $(\alpha, \beta) \rightarrow v_{n}(., . ; \alpha, \beta)$ is continuous from $S$ to $L^{1}\left(Q, R^{n}\right)$. 
(b) $v_{n}(x, y ; \alpha, \beta) \in F\left(x, y, u_{n}(x, y ; \alpha, \beta)\right)$ for any $(\alpha, \beta) \in \mathbf{S}$ and a.e. $(x, y) \in Q$.

(c) $\left\|v_{n}(x, y ; \alpha, \beta)-v_{n-1}(x, y ; \alpha, \beta)\right\| \leqq L \sigma_{n}(\alpha, \beta)(x, y)$ a.e. in $Q$.

(d) $u_{n}(x, y ; \alpha, \beta)=u_{0}(x, y ; \alpha, \beta)+\int_{0}^{x} \int_{0}^{y} v_{n-1}(\xi, \eta ; \alpha, \beta) d \xi d \eta$

Suppose we have constructed $v_{1}, \ldots, v_{n}$ and $u_{1}, \ldots, u_{n}$ satisfying (a)-(d). Then define

$$
u_{n+1}(x, y ; \alpha, \beta)=u_{0}(x, y ; \alpha, \beta)+\int_{0}^{x} \int_{0}^{y} v_{n}(\xi, \eta ; \alpha, \beta) d \xi d \eta
$$

Let $(x, y) \in Q \backslash\{(0,0)\}$. Using (c) we have

$$
\begin{aligned}
& \left\|u_{n+1}(x, y ; \alpha, \beta)-u_{n}(x, y ; \alpha, \beta)\right\| \leqq \int_{0}^{x} \int_{0}^{y}\left\|v_{n}(\xi, \eta ; \alpha, \beta)-v_{n-1}(\xi, \eta ; \alpha, \beta)\right\| d \xi d \eta \\
& \leqq \\
& \quad L \int_{0}^{x} \int_{0}^{y} \sigma_{n}(\alpha, \beta)(\xi, \eta) d \xi d \eta=L^{n} \int_{0}^{x} \int_{0}^{y} \sigma(\alpha, \beta)(\xi, \eta)\left(\int_{\xi}^{x} \frac{(x-u)^{n-1}}{(n-1) !} d u \int_{\eta}^{y} \frac{(y-v)^{n-1}}{(n-1) !} d v\right) d \xi d \eta \\
& \quad+L^{n}\left(\sum_{i=0}^{n} \varepsilon_{i}\right) \int_{0}^{x} \int_{0}^{y} \frac{(\xi-\eta)^{n}}{n !} d \xi d \eta=L^{n} \int_{0}^{x} \int_{0}^{y} \frac{(x-\xi)^{n}}{n !} \frac{(y-\eta)^{n}}{n !} \sigma(\alpha, \beta)(\xi, \eta) d \xi d \eta \\
& \quad+\frac{L^{n}}{n !}\left(\sum_{i=0}^{n} \varepsilon_{i}\right) \frac{(x+y)^{n+2}-x^{n+2}-y^{n+2}}{(n+1)(n+2)} \leqq L^{n}\left[\int_{0}^{x} \int_{0}^{y} \frac{(x-\xi)^{n}}{n !} \frac{(y-\eta)^{n}}{n !} \sigma(\alpha, \beta)(\xi, \eta) d \xi d \eta\right. \\
& \left.\quad+\left(\sum_{i=0}^{n} \varepsilon_{i}\right) \frac{(x+y)^{n+1}}{(n+1) !}\right]<\sigma_{n+1}(\alpha, \beta)(x, y),
\end{aligned}
$$

Then, by virtue of (2.4) and of the assumption $\left(\mathrm{H}_{2}\right)$, it follows that

$$
\begin{aligned}
d\left(v_{n}(x, y ; \alpha, \beta), F\left(x, y, u_{n+1}(x, y ; \alpha, \beta)\right)\right) & \leqq L\left\|u_{n+1}(x, y ; \alpha, \beta)-u_{n}(x, y ; \alpha, \beta)\right\| \\
& <L \sigma_{n+1}(\alpha, \beta)(x, y),
\end{aligned}
$$

Since $\sigma$ is continuous from $S$ to $L^{1}(Q, R)$, by (2.3) it follows that also $\sigma_{n}$ is continuous from $S$ to $L^{1}(Q, R)$. Therefore, by $(2.5)$ and Lemma 1 , we have that the multivalued map $G_{n+1}$ defined by

$$
G_{n+1}(\alpha, \beta)=\left\{v \in L^{1}(Q, X): v(x, y) \in F\left(x, y, u_{n+1}(x, y ; \alpha, \beta)\right) \text { a.e. in } Q\right\}
$$

is 1.s.c. from $\mathbf{S}$ to $\mathscr{D}$. Moreover, by (2.5), it follows that 


$$
H_{n+1}(\alpha, \beta)=c l\left\{v \in G_{n+1}(\alpha, \beta):\left\|v(x, y)-v_{n}(x, y ; \alpha, \beta)\right\|<L \sigma_{n+1}(\alpha, \beta)(x, y) \text { a.e. in } Q\right\}
$$

is nonempty. Then, by Lemma 2 , there exists $h_{n+1}: \mathbf{S} \rightarrow L^{1}\left(Q, R^{n}\right)$ a continuous selection of $H_{n+1}$. Set $v_{n+1}(x, y ; \alpha, \beta)=h_{n+1}(\alpha, \beta)(x, y)$ and observe that $v_{n+1}$ satisfies the properties (a)-(d). By (c) and the computations in (2.4) it follows that

$$
\left\|v_{n}(., ; \alpha, \beta)-v_{n-1}(., . ; \alpha, \beta)\right\|_{1} \leqq \frac{L^{n}}{n !}\|\sigma(\alpha, \beta)\|_{1}+\varepsilon \frac{[2 L]^{n}}{n !} .
$$

and

$$
\begin{aligned}
\left\|u_{n+1}(., . ; \alpha, \beta)-u_{n}(., . ; \alpha, \beta)\right\|_{\infty} & \leqq \\
& \leqq v_{n+1}(., . ; \alpha, \beta)-v_{n-1}(., . ; \alpha, \beta) \|_{1} \\
& \frac{L^{n}}{n !}\|\sigma(\alpha, \beta)\|_{1}+\varepsilon \frac{[2 L]^{n}}{n !} .
\end{aligned}
$$

Therefore $\left\{u_{n}(., . ; \alpha, \beta)\right\}_{n \in N}$ and $\left\{v_{n}(., . ; \alpha, \beta\}_{n \in N}\right.$ are Cauchy sequences in $C\left(Q, R^{n}\right)$ and $L^{1}\left(Q, R^{n}\right)$, respectively. Moreover since $(\alpha, \beta) \rightarrow\|\sigma(\alpha, \beta)\|_{1}$ is continuous, it is locally bounded; hence the Cauchy condition is satisfied locally uniformly with respect to $(\alpha, \beta)$. Let $u(., . ; \alpha, \beta) \in C\left(Q, R^{n}\right)$ and $v(., . ; \alpha, \beta) \in L^{1}\left(Q, R^{n}\right)$ be the limit of $\left\{u_{n}(x, y ; \alpha, \beta)\right\}$ and $\left\{v_{n}(., . ; \alpha, \beta)\right\}$ respectively. Then $(\alpha, \beta) \rightarrow u(., . ; \alpha, \beta)$ is continuous from $\mathbf{S}$ to $C(Q, X)$ and $(\alpha, \beta) \rightarrow v(.,, ; \alpha, \beta)$ is continuous from $S$ to $L^{1}\left(Q, R^{n}\right)$. Letting $n \rightarrow \infty$ in (d) we obtain that

$$
u(x, y ; \alpha, \beta)=u_{0}(x, y ; \alpha, \beta)+\int_{0}^{x} \int_{0}^{y} v(\xi, \eta ; \alpha, \beta) d \xi d \eta \quad \text { for any }(x, y) \in Q .
$$

Furthermore, since

$$
d\left(v_{n}(x, y ; \alpha, \beta), F(x, y, u(x, y ; \alpha, \beta))\right) \leqq L\left\|u_{n+1}(x, y ; \alpha, \beta)-u(x, y ; \alpha, \beta)\right\|
$$

and $F$ has closed values, letting $n \rightarrow \infty$ we have

$$
v(x, y ; \alpha, \beta) \in F(x, y, u(x, y ; \alpha, \beta)) \quad \text { a.e. in } Q .
$$

By (2.8) and (2.9) it follows that $u(., . ; \alpha, \beta)$ is a solution of $\left(D_{\alpha \beta}\right)$, which completes the proof.

Remark 1. Theorem 1 remains true (with the same proof) if $R^{n}$ is replaced by a separable Banach space $X$ and $F$ is a multifunction from $Q \times X$ to the closed nonempty subsets of $X$ satisfying the assumptions $\left(H_{1}\right)-\left(H_{3}\right)$.

Remark 2. If the assumption $\left(\mathrm{H}_{2}\right)$ is relaxed, allowing $F$ to be merely continuous then the conclusion of the theorem is in general no longer true. To see this consider the Darboux problem 
$\left(D_{\alpha, \beta}\right)$

$$
u_{x y}=\sqrt[3]{u}, \quad u(x, 0)=\alpha(x), \quad u(0, y)=\beta(y), \quad(x, y) \in Q
$$

Remark that $f(u)=\sqrt[3]{u}$ is continuous but not Lipschitzean in a neighbourhood of 0 and, for $\alpha_{0}(x)=0=\beta_{0}(y)$, the problem $\left(D_{\alpha_{0}, \beta_{0}}\right)$ admits as solutions:

$$
u_{0}^{+}(x, y)=\left(\frac{2}{3}\right)^{3} x^{3 / 2} y^{3 / 2} \text { and } u_{0}^{-}(x, y)=-\left(\frac{2}{3}\right)^{3} x^{3 / 2} y^{3 / 2}
$$

Let

$$
\alpha_{n}^{+}(x)=\left(\frac{2}{3 \sqrt{n}}\right)^{3} x^{3 / 2}, \quad \alpha_{n}^{-}(x)=-\left(\frac{2}{3 \sqrt{n}}\right)^{3} x^{3 / 2}, \quad \beta_{n}^{+}(y)=0=\beta_{n}^{-}(y)
$$

Then

$$
\left(\alpha_{n}^{+}, \beta_{n}^{+}\right),\left(\alpha_{n}^{-}, \beta_{n}^{-}\right) \in \mathbf{S} \text { and }\left\|\left(\alpha_{n}^{+}, \beta_{n}^{+}\right)\right\|=\left\|\left(\alpha_{n}^{-}, \beta_{n}^{-}\right)\right\|=\left(\frac{2}{3 \sqrt{n}}\right)^{3}
$$

therefore $\left(\alpha_{n}^{+}, \beta_{n}^{+}\right)$and $\left(\alpha_{n}^{-}, \beta_{n}^{-}\right)$converge to $\left(\alpha_{0}, \beta_{0}\right)=(0,0)$ in the space $S$.

On the other hand the unique solution of the Darboux problem $\left(D_{a_{n}^{+}, \beta_{n}^{+}}\right)$(resp. of $\left.\left(D_{\alpha_{n}^{-}, \beta_{n}^{-}}\right)\right)$is given by

$$
\begin{gathered}
u_{n}^{+}(x, y)=\left(\frac{2}{3}\right)^{3} x^{3 / 2}\left(\frac{1}{n}+y\right)^{3 / 2} \\
\left(\text { resp. } u_{n}^{-}(x, y)=-\left(\frac{2}{3}\right)^{3} x^{3 / 2}\left(\frac{1}{n}+y\right)^{3 / 2}\right)
\end{gathered}
$$

which for $n \rightarrow \infty$ converges to $u_{0}^{+}$(resp. $u_{0}^{-}$).

Suppose that there exists $r: S \rightarrow C(Q, X)$ a continuous selection of the solution map $(\alpha, \beta) \rightarrow \mathrm{T}(\alpha, \beta)$. Then, for $n \rightarrow \infty$, we have that $r\left(\left(\alpha_{n}^{+}, \beta_{n}^{+}\right)\right)=u_{n}^{+}$converges to $u_{0}^{+}$and $r\left(\left(\alpha_{n}^{-}, \beta_{n}^{-}\right)\right)=u_{n}^{-}$converges to $u_{0}^{-}$. This is a contradiction to the continuity of $r$.

\section{REFERENCES}

1. J. P. Aubin and A. Cellina, Differential Inclusions (Springer-Verlag, Berlin, 1984).

2. A. Bressan and G. Colombo, Extensions and selections of maps with decomposable values, Studia Math. 90 (1988), 69-86.

3. M. Cinquini-Cibrario and S. Cinquini, Equazioni a Derivate Parziali di Tipo Iperbolico Parziali (Edizioni Cremonese, Roma, 1964).

4. R. M. Colombo, A. Fryszkowski, T. Rzezuchowski and V. Staicu, Continuous selection of solutions sets of Lipschitzean differential inclusions, Funkcial. Ekvac. 34 (1991), 321-330. 
5. F. S. De Blasi and J. MyjaK, On the set of solutions of a differential inclusion, Bull. Inst. Math. Acad. Sinica. 14 (1986), 271-275.

6. F. S. De Blasi and J. Myjak, On the structure of the set of solutions of the Darboux problem of hyperbolic equations, Proc. Edinburgh Math. Soc. 29 (1986), 7-14.

7. A. F. Filippov, Classical solutions of differential equations with multivalued right hand side, SIAM J. Control 5 (1967), 609-621.

8. A. Fryszkowski, Continuous selections for a class of nonconvex multivalued maps, Studia Math. 76 (1983), 163-174.

9. F. Hial and H. UMegaKi, Integrals, conditions expectations and martingales of multivalued functions, J. Multivariate Anal. 7 (1971), 149-182.

10. S. Marano, Generalized solutions of partial differential inclusions depending on a parameter, Rend. Acad. Naz. Sci. XL Mem. Mat. 107 (1989), 281-295.

11. G. Teodoru, Le problem de Darboux pour une equation aux derivees partielles multivoque, An. Stiint. Univ. "Al. I. Cuza” lasi Sect. I a Mat. 31 (1985), 173-176.

12. G. Teodoru, Sur le problem de Darboux pour l'equation $\partial^{2} z / \partial x \partial y \in F(x, y, z)$, An. Stiint. Univ. “Al. I. Cuza” Iasi Sect. I a Mat. 32 (1986), 41-49.

13. G. Teodoru, Continuous selections for multifunctions satisfying Carathéodory type conditions. The Darboux problem associated to a multivalued equation, Proc. Itinerant Seminar on Functional Equations, Approximation and Convexity, Faculty of Mathematics, Cluj-Napoca, Preprint 6 (1987), 281-286.

International School for Advanced Studies

Strada Costiera II.

34014 TRIESTE

ITALY 